\title{
Critical points of invariant functions on closed orientable surfaces
}

\author{
G. Gromadzki · J. Jezierski · W. Marzantowicz
}

Received: 4 April 2013 / Accepted: 2 April 2014 / Published online: 6 May 2014

(C) The Author(s) 2014. This article is published with open access at Springerlink.com

\begin{abstract}
The relationship between critical points of equivariant functions and topological invariants of an equivariant action on closed manifold is an interesting problem. In this paper, we study this relationship for orientation-preserving actions of finite groups $G$ on a closed orientable surfaces. We give an elementary, but detailed, description of the behaviour of the gradient field of an equivariant $C^{1}$-function, we present an elementary, differential, proof of the Riemann-Hurwitz formula and we construct invariant $C^{1}$-functions with the minimal number of critical orbits. These lead us to show that, with a few exceptions, the equivariant Lusternik-Schnirelmann category of a closed orientable topological surface equals the number of singular orbits of the action.
\end{abstract}

Keywords Riemann surfaces - Finite group action - Equivariant LusternikSchnirelmann category $\cdot$ Riemann-Hurwitz ramification formula $\cdot$ Invariant function · Critical orbits

G. Gromadzki supported by NCN 2012/05/B/ST1/02171 and by Max-Planck Mathematical Institute in Bonn, J. Jezierski supported NN 201 373236, and W. Marzantowicz supported by NN 201 373236, and NCN 2011/03/B/ST1/04533.

G. Gromadzki

Institute of Mathematics, Gdańsk University, Wita Stwosza 57, 80-952 Gdańsk, Poland e-mail: grom@mat.ug.edu.pl

J. Jezierski

Institute of Applied Mathematics, University of Agriculture, ul. Nowoursynowska 166, 02-787 Warsaw, Poland

e-mail: jerzy_jezierski@sggw.pl

W. Marzantowicz $(\varangle)$

Faculty of Mathematics and Computer Science, Adam Mickiewicz University of Poznań, Umultowska 87, 61-614 Poznań, Poland

e-mail:marzan@amu.edu.pl 
Mathematics Subject Classification (1991) Primary 55M30 · 55M35 · Secondary 30F10

\section{Introduction}

Throughout the whole paper we shall, usually, use the letter $X$ to denote a topological space and $M$ when this space is a manifold with a differential structure. A compact Riemann surface, which is understood as a two-dimensional compact closed surface $M$ with a Riemannian metric and thus as a surface equipped with a holomorphic structure will be denoted, by $\mathcal{S}$. We shall see that, without loss of generality, a finite orientationpreserving faithfull action of a finite group $G$ on a closed orientable topological surface $X$ can be assumed to be holomorphic for some Riemann surface $\mathcal{S}$ homeomorphic to $X$.

In the first part of this paper, we study a behaviour of $G$-invariant functions, showing a correspondence between $G$-invariant $C^{1}$-functions on the surface $M$ and $C^{1}$ functions on its orbit space $M^{\prime}=M / G$. Note that, since our paper concerns surfaces, all such orbit spaces are manifolds, as follows from Theorem 2.4. We derive a formula for the index of the gradient at a critical point of an invariant function (Lemma 3.5 and Corollary 3.7) which allows us to give a new, differential, proof of the RiemannHurwitz formula. It is worth to be pointed out that all of the arguments used are completely elementary.

Calculating the equivariant Lusternik-Schnirelmann category of a space $X$ with an action of compact Lie group $G$ is an interesting problem. In the second part of this work, we give a complete characterization of equivariant category for an orientationpreserving action of a finite group $G$ on a closed orientable topological surface $X$. The main result (Theorem 4.11) says that, apart from some special cases, the equivariant Lusternik-Schnirelmann category of such a surface is equal to the number of singular orbits, by which we mean the orbits of lengths smaller than the order of $G$. Consequently, given a surface $X$ of genus $g=g(X)$, the problem of finding actions of a finite group $G$ with a given equivariant Lusternik-Schnirelmann category is equivalent to the problem of constructing actions of $G$ with prescribed number of periods. For a cyclic group $G=\mathrm{Z}_{N}$, a more general problem of finding necessary and sufficient conditions for the existence of such action on a surface with prescribed set of periods and given genus was solved in $[7,14]$. This relationship was a principal motivation for the authors while working on this subject. Nevertheless, the starting point for our studies of equivariant category of a surface, was the paper by Colman [4].

The classical Lusternik-Schnirelmann category gives a lower estimate for the number of critical points of any $C^{1}$-function. Its equivariant version gives a similar estimate for an equivariant $C^{1}$-function $f: M \rightarrow \mathbb{R}$ (cf. [1,3,11]). Consequently, this notion has also important applications in studies of multiplicities of nonlinear variational problems with symmetry (see [1] for a review of results), which can be seen as another justification of our study.

\section{Orientation-preserving actions on surfaces}

We refer the reader to the papers $[1,4,11]$ for the basic definitions and principal facts concerning equivariant category. Let $G$ be a compact Lie group acting on a manifold 
$M$. An invariant subspace $U$ of $M$ is called $G$-categorical if it is contractible through a $G$-equivariant homotopy by which we understand homotopy $F$ which is $G$-invariant on each level $F_{t}$.

The equivariant category of a closed $G$-invariant subset $A$ of a topological space $X$, denoted by cat ${ }_{G}(A ; X)$, is the smallest possible number of $G$-categorical, within the whole space $X$ and not only in $A$, invariant open subsets required to cover $A$ and it is defined to be infinity $\infty$ if no such covering exists. If $A=X$, then we simply denote $\operatorname{cat}_{G}(A ; X)$ by $\operatorname{cat}_{G}(X)$ and call it the equivariant category of $X$. Clearly, the equivariant category is invariant under equivariant homeomorphisms, and coincides with the classical Lusternik-Schnirelmann category for the case of trivial action.

In this paper, we give a complete characterization of the equivariant category for any orientation-preserving action of finite Lie groups on compact orientable unbordered surfaces. We begin with a classical and well-known observation, that for surfaces with negative Euler characteristic, the case of finite group action studied here is actually the only possible one.

Proposition 2.1 Let $M$ be a closed compact orientable smooth manifold of dimension 2 with a negative Euler characteristic $\chi=\chi(M)$, i.e. a Riemann surface $\mathcal{S}$ of genus $\mathrm{g}=1-\chi(M) / 2 \geq 2$. Then, there is no smooth, nontrivial action of a compact connected Lie group $G$ of nonzero dimension on $M$. Consequently, any compact Lie group acting smoothly and nontrivially on $M$ must be finite.

Proof Let $\mathbb{T}=\mathbb{T}^{d} \subset G$ be a maximal torus of $G$. Suppose that the set $M^{\mathbb{T}}$ of points fixed by $\mathbb{T}$ in $M$ is equal to $M$, which simply means that the action of $\mathbb{T}$ is trivial. Also, any $g \in G$ is conjugate to an element $t \in \mathbb{T}$, say $g=g^{\prime} t g^{\prime-1}$. Then, for $x \in X$, $g x=\left(g^{\prime} t^{\prime-1}\right) x=g^{\prime}\left(t\left(g^{\prime-1} x\right)\right)=g^{\prime}\left(g^{\prime-1} x\right)=\left(g^{\prime} g^{\prime-1}\right) x=e x=x$ and so the action of the whole group $G$ on $M$ must be trivial as well.

We are left with the case when the action of $\mathbb{T}$ is nontrivial. Let $\varphi: G \rightarrow \operatorname{Homeo}(M)$ be the homeomorphism induced by this action. The latter means that $\operatorname{ker} \varphi \neq \mathbb{T}$ and so the quotient group $\mathbb{T} / \operatorname{ker} \varphi$ is embedded into $\mathrm{Homeo}^{+}(M)$. Since any proper subgroup of

$$
\mathbb{T} \cong \mathbb{S}_{1}^{1} \times \cdots \times \mathbb{S}_{d}^{1}
$$

is equal, up to an isomorphism of $\mathbb{T}$, to

$$
\mathbb{S}_{1}^{1} \times \cdots \times \mathbb{S}_{k}^{1} \times \mathbb{Z}_{n_{1}} \times \cdots \times \mathbb{Z}_{n_{d-k}}
$$

with $k<d$, there exists an index $i$ for which $\operatorname{ker} \varphi \cap \mathbb{S}_{i}^{1}=\mathbb{Z}_{n_{i}}$. Consequently, $\mathbb{T} / \operatorname{ker} \varphi$ contains $\mathbb{S}^{1} / \mathbb{Z}_{n_{i}} \cong \mathbb{S}^{1}$. This implies that the group $\operatorname{Homeo}(M)$ contains cyclic subgroups of arbitrarily large order, which is impossible for a compact surface of genus $g \geq 2$ due to the classical Hurwitz theorem, which we present below for a reader's convenience (see also classical result of Wimann [17] for the precise bound on the order in this case).

Remark 2.2 If $M$ is the sphere $\mathbb{S}^{2}$ with the Riemannian metric induced by the canonical embedding in $\mathbb{R}^{3}$ or a torus $\mathbb{T}^{2}=\mathbb{R}^{2} / \mathbb{Z}^{2}$ with the flat Riemannian metric, then the 
group of isometries is infinite and contains $\mathbb{S}^{1}$ or $\mathbb{T}^{2}$, respectively. Therefore, for these surfaces, we have to make an additional assumption, that the group acting is finite.

Remark 2.3 First remind that every topological closed surface $X$ posseses only one differential structure $M$. If $X$ is a closed surface of negative Euler characteristic then, due to the mentioned Hurwitz [8] theorem, every finite group of self-homeomorphisms can be represented as a subgroup of the group of conformal automorphisms of a Riemann surface $\mathcal{S}$ homeomorphic to $X$. Consequently, we may assume that every effective orientation-preserving action of a finite group $G$ on a compact orientable surface $X$ by which we mean any faithful representation of $G$ in $\mathrm{Homeo}^{+}(X)$, can be realized by conformal maps, i.e. by an embedding of $G$ into $\operatorname{Aut}(\mathcal{S})$ for such $\mathcal{S}$. This gives a first well-known upper bound for the order of a group $G$ acting effectively on $X$, since the second part of the mentioned theorem of Hurwitz says that a group of automorphisms $G$ of a compact Riemann surface $\mathcal{S}$ of topological genus g has at most 84( $g-1)$ elements. However, the statement of the Hurwitz theorem holds also for an orientation-preserving action of a finite group on the sphere and torus. Indeed, for the sphere, Brouwer, Kerekjarto and Eilenberg showed sequentially that every action of a finite group $G$ is equivalent to an orthogonal action (see [5] for references). Also, the case of torus can be studied in the same way, as it was observed by Edmonds [5] though, some people consider it as a mathematical folklore only.

These lead to the following characterization of actions.

Theorem 2.4 (Geometrization of action) Given a finite group $G$ of orientationpreserving homeomorphisms of a compact topological surface $X$ of an arbitrary genus $\mathrm{g}$, there is a complex structure on $X$ with respect to which $G$ is a group of conformal automorphisms of resulting Riemannian surface $\mathcal{S}$, thus diffeomorphisms of corresponding manifold $M$. Moreover, the orbit space $M^{\prime}=M / G$ is a compact (smooth!) surface of genus $\mathrm{g}^{\prime} \leq \mathrm{g}$ which carries the additional structure of an orbifold. Furthermore, if $\mathrm{g}>1$ then $\mathrm{g}^{\prime}<\mathrm{g}$ as follows from the Riemann-Hurwitz formula 5.

Proof For the first part of the statement, we refer to [5]. The second is an easy consequence, but we would like to make a comment on it. Since the action is holomorphic, thus smooth, in some $G_{x}$-invariant neighbourhood of any point $x \in X$ it is $G_{x^{-}}$equivariantly diffeomorphic to an orthogonal action on two discs $\mathbb{D}^{2}$ of the isotropy group $G_{x}$ of $x$. By the Proposition 2.6, $G_{x}=\mathbb{Z}_{m}$. The action of $G$ is free outside a finite union of discs corresponding to all $x$ with $G_{x} \neq e$. This shows that the orbit space is a smooth manifold, thus a surface, because at every $x$ with $G_{x}=Z_{m} \neq e$, the orbit space $\mathbb{D}^{\prime}=\mathbb{D}^{2} / \mathbb{Z}_{m}$ is diffeomorphic to $\mathbb{D}^{2}$ (however, the projection map $\pi: \mathbb{D}^{2} \rightarrow \mathbb{D}^{\prime}=\mathbb{D}^{2} / \mathbb{Z}_{m}$ is a branched cover then). Secondly, $\mathrm{g}^{\prime}<\mathrm{g}$ follows from the Riemann-Hurwitz formula (cf. [6], see also equality).

Remark 2.5 We should point out here, that for any orientation-preserving action of a finite group $G$ on a closed surface $X$ of genus $g$, the statement of Theorem 2.4 is equivalent to one of the following (cf. [5]):

1. there is a differential structure and a Riemaniann metric of constant curvature on $X$, so that $G$ is a group of isometries of the resulting manifold $M$; 
2. the universal covering $\tilde{M}$ of $M$ can be identified with the Euclidean sphere $\mathbb{S}^{2}$, the Euclidean plane $\mathbb{R}^{2}$ or the hyperbolic upper half-plane $\mathcal{H}$ if $g=0,1$ or $\geq 2$, respectively, in such a way that all the lifts of all the elements of $G$ to $\tilde{M}$ correspond to a group of isometries of $\tilde{M}$.

Next, we remind a classical fact, which allows us to restrict ourselves to the cyclic groups (see [12, Prop 3.1]) in our study of the sets of points fixed by orientationpreserving actions of finite groups on compact orientable surfaces. As before, the fixed point set of a $G$-space, with respect to a subgroup $H$ of $G$, is denoted by $X^{H}$.

Proposition 2.6 Let $G$ be a group of holomorphic automorphisms of a compact Riemann surface $M=\mathcal{S}$ and let $M^{H} \neq \varnothing$ for a subgroup $H \subset G$. Then, $H$ is cyclic.

Remark 2.7 The above is only the necessary condition. The sufficient condition for $M^{H}$ to be nonempty, for a cyclic group $H \subset G$, is that a lifting of $H$ to $\tilde{M}$ in Remark 2.5 (2) contains an elliptic element of order being equal to the order of $H$ (cf. $[9,10]$ ).

\section{Critical points of invariant functions on compact surfaces}

In this section, we shall study critical points of a $G$-invariant function $f$ on a compact surface with a holomorphic action of a finite group $G$.

\subsection{Properties of the gradient field of an invariant function}

Let $M$ be a closed two-dimensional manifold, i.e. a compact surface and let $f: M \rightarrow$ $\mathbb{R}$ be a $C^{1}$-function. Then, the gradient of $f$ defines a continuous vector field

$$
\mathfrak{X}: M \rightarrow T M ; \quad \mathfrak{X}(x)=\nabla(f)(x) .
$$

A point $x \in M$ is called a critical point of a $C^{1}$-function $f: M \rightarrow \mathbb{R}$ if the gradient vanishes at $x$, i.e. $\nabla f(x)=0$.

By definition, the set of zeros $\operatorname{Zer}(\mathfrak{X})$ is equal to the set $\operatorname{Crit}(f)$ of critical points of $f$.

Let $M$ be a compact closed manifold, let $f: M \rightarrow \mathbb{R}$ be a $C^{1}$-function with isolated critical points and let $\mathfrak{X}(x)=\nabla(f)(x): M \rightarrow T M$ be the associated gradient vector field.

Recall that the local index at isolated zero $x_{0}$ of a vector field $\mathfrak{X}: M \rightarrow T M$ is the degree of the map

$$
x \mapsto \frac{\mathfrak{X}(x)}{|\mathfrak{X}(x)|}
$$

mapping a small sphere $\mathbb{S}_{\mathcal{E}}\left(x_{0}\right) \subset M$ into the unit sphere $\mathbb{S}^{1}$ in the tangent space at $x_{0}$. Equivalently, it is equal to the degree of a map of pairs $\mathfrak{X}:\left(\mathbb{D}_{\epsilon}^{2}, \mathbb{D}_{\epsilon}^{2} \backslash\{0\}\right) \rightarrow$ $\left(\mathbb{R}^{2}, \mathbb{R}^{2} \backslash\{0\}\right)$ induced by $\mathfrak{X}$ in local coordinates. 
The classical Hopf formula, applied to the field $\nabla(f)(x)$, says that

$$
\sum_{x_{i} \in \operatorname{Crit}(f)} \operatorname{ind}(\nabla(f))\left(x_{i}\right)=\chi(M),
$$

where $\chi(M)$ is the Euler characteristic of $M$. Recall that for a surface, we have $\chi(M)=2-2 \mathrm{~g}(M)$.

Remark 3.1 Let $M$ be a manifold with a smooth action of a finite group $G$. Note that if $f: M \rightarrow \mathbb{R}$ is a smooth $C^{1}$ function with isolated critical points, then

$$
\tilde{f}: M \rightarrow \mathbb{R} \quad \tilde{f}(x):=\frac{1}{|G|} \sum f\left(g^{-1} x\right)
$$

is $G$-invariant function with isolated, thus finite in amount, critical points.

In the next, we present the correspondence between the local germs of $C^{1}$-functions at a singular point $x_{0}$ of a surface and its image $x_{0}^{\prime}$ in the orbit space $M^{\prime}=M / G$. First notice that, locally, the action of $G$ near zero is equivariantly diffeomorphic to the tubular neighbourhood $G \times{ }_{G_{x_{0}}} D_{\varepsilon}$, where $G_{x_{0}}$ is the isotropy group of $x_{0}$ and $D_{\varepsilon}$ is a small disc at 0 in the tangent space at $x_{0}$. Consequently, for some open invariant set $U$, containing the orbit $G x_{0}$, we have

$$
U / G \equiv \mathbb{D}_{\varepsilon} / G_{x_{0}} \equiv \mathbb{D}_{\varepsilon} / Z_{m}
$$

since each isotropy group is cyclic (Proposition 2.6).

Theorem 3.2 Suppose that $G$ is a finite group acting preserving orientation on a closed compact surface $M$ with $M^{\prime}=M / G$ and $\pi: M \rightarrow M^{\prime}$ the canonical projection. Then,

1. Every $C^{1}$-function $f^{\prime}: M^{\prime} \rightarrow \mathbb{R}$ defines an invariant $C^{1}$-function $f:=f^{\prime} \circ \pi$ such that all points of all singular orbits of the action are critical points of $f$.

2. Conversely, for an invariant $C^{1}$-function $f: M \rightarrow \mathbb{R}$, each singular point of the action is a critical point.

3. Furthermore, any $G$-invariant $C^{1}$-function $f: M \rightarrow \mathbb{R}$ defines the unique $C^{0}$ function $f^{\prime}: M^{\prime} \rightarrow \mathbb{R}$, such that $f=f^{\prime} \circ \pi$.

Moreover, if at any singular point of action $x$ with isotropy group $G_{x} \equiv \mathbb{Z}_{m}$ the function $f$ is of class $C^{m}$ and

$$
D f(x)=D^{2} f(x)=\cdots=D^{m} f(x)=0
$$

then $f^{\prime}=f / G$ is a $C^{1}$-function and the image $x^{\prime}=\pi(x)$ of any singular point $x$ is a critical point of $f^{\prime}$.

Proof By Theorem 2.4, we can assume that $M=\mathcal{S}$ is a Riemann surface and $G \subset$ $\operatorname{Aut}(\mathcal{S})$, thus $\operatorname{Sing}(M)=\operatorname{Sing}(\mathcal{S})$. 
To prove (1), it is enough to notice that $D \pi$ is the zero map at each singular point. To get (2), observe that for an invariant $C^{1}$-function $f: M \rightarrow \mathbb{R}$, a singular point is critical. Indeed, $\nabla f: M \rightarrow T M$ is an equivariant vector field and thus $\nabla(f)(g x)=D g(x)[\nabla f(x)]$, where $D g(x)$ is the derivative of $g$ at $x$. This gives $\nabla f(x)=\nabla f(g x)=D g(x) \nabla f(x)$ for every $g \in G_{x}$. Therefore, since $\left(T_{x} M\right)^{G_{x}}=$ 0 , we have that $G_{x} \neq e$ implies $\nabla f(x)=0$.

Finally, concerning the proof of (3), observe that $f$ defines a continuous function $f^{\prime}: M^{\prime} \rightarrow \mathbb{R}$ given by $f^{\prime}\left(x^{\prime}\right):=f(x)$, where $x^{\prime}=\pi(x)$. For, it is sufficient to show that $f^{\prime}$ is a $C^{1}$-function at $x^{\prime}=\pi(x)$ for every singular point $x$, as $\pi: S \backslash \operatorname{Sing}(M) \rightarrow$ $M^{\prime} \backslash \pi(\operatorname{Sing}(M))$ is a finite regular covering. The statement of the Theorem 3.2 follows from the lemma below.

Lemma 3.3 Let a cyclic group $G=\mathbb{Z}_{m}$ act orthogonally and semi-freely on $\mathbb{D}^{2} \subset \mathbb{C}$, with 0 being the only fixed point. Let $f: \mathbb{D}^{2} \rightarrow \mathbb{R}$ be a $C^{m}, G$-invariant function with a critical point at 0 such that $D f(0)=D^{2} f(0)=\cdots=D^{m} f(0)=0$. Then, $\mathbb{D}^{2} / G$ is diffeomorphic to $\mathbb{D}^{2}$ and $\pi: \mathbb{D}^{2} \rightarrow \mathbb{D}^{2} / G$ is a branched covering with a group of deck transformation generated by $z \mapsto \varepsilon z, \varepsilon$ the rotation by the angle $2 \pi / \mathrm{m}$. Moreover, $f$ induces a $C^{1}$-function $f^{\prime}$ on $\mathbb{D}^{2} / G$, which has an isolated critical point at 0 and $f=f^{\prime} \circ \pi$.

Proof Since the action of $G$ is orthogonal and semi-free, an element $g \in \mathbb{Z}_{m}$, represented by $1 \leq k \leq m-1$, acts as the linear map $\exp (2 \pi \imath k) \in \mathrm{U}(1)=\mathrm{SO}(2) \subset \mathrm{O}(2)$. Then, obviously, $\mathbb{D}^{2} / G=\mathbb{D}^{2}$ and $\pi: \mathbb{D}^{2} \rightarrow \mathbb{D}^{2} / G$ is equal to $\pi(z)=z^{m}$ in the complex coordinates of $\mathbb{D}^{2}$.

Therefore, it remains to show that $f^{\prime}=f / G$ is $C^{1}$ at 0 . Adding a constant if necessary, we can assume that $f(0)=0$. Let $z_{n}^{\prime} \rightarrow 0, z_{n}^{\prime} \neq 0$, be an arbitrary sequence. We choose a point $z_{n} \in \pi^{-1}\left(z_{n}^{\prime}\right)$ for each $n \in \mathbb{N}$. Let us notice that then $\left|z_{n}\right|^{m}=\left|z_{n}^{\prime}\right|$. By the definition, $f^{\prime}\left(z_{n}^{\prime}\right)=f\left(z_{n}\right)$. Note that by our assumption $f(z)=o\left(|z|^{m}\right)$, i.e. $|f(z)| /|z|^{m} \rightarrow 0$ if $|z| \rightarrow 0$. Consequently,

$$
\lim _{n \rightarrow \infty} \frac{f^{\prime}\left(z_{n}^{\prime}\right)-f^{\prime}(0)}{\left|z_{n}^{\prime}\right|}=\lim _{n \rightarrow \infty} \frac{f\left(z_{n}\right)}{\left|z_{n}\right|^{m}}=0,
$$

which shows that at $0 f^{\prime}$ has the derivative $D f^{\prime}(0)=\nabla\left(f^{\prime}\right)(0)=0$. To prove that $f^{\prime}$ is $C^{1}$, we need to show that $D f^{\prime}\left(z_{n}^{\prime}\right) \rightarrow D f^{\prime}(0)=0$ for any sequence $z_{n}^{\prime} \rightarrow 0$. Since we may assume that $z_{n}$ is regular, $\pi$ is a covering near $z_{n}$ and $D f\left(z_{n}\right)=D f^{\prime}\left(z_{n}^{\prime}\right)$. Now $D f^{\prime}\left(z_{n}^{\prime}\right)=D f\left(z_{n}\right) \rightarrow D f(0)=0$ which proves that $f^{\prime}$ is $C^{1}$.

Corollary 3.4 Let $f: M \rightarrow \mathbb{R}$ be a $C^{\infty}, G$-invariant function. Then, $f^{\prime}=f / G$ is also a $C^{\infty}$-function provided $f$ is flat at every singular point $x$ of the action. Moreover, $f^{\prime}$ is also flat at $\pi(x)$.

Proof Recall that $f$ is flat at $x_{0}$ means that $D^{k} f\left(x_{0}\right)=0$ for every $k \geq 1$. This yields that $\left|D^{k} f(x)-D^{k} f\left(x_{0}\right)\right|=\left|D^{k} f(x)\right|=o\left(\left|x-x_{0}\right|^{n}\right)$ for every $k, n \geq 1$. We have already shown that $D f^{\prime}\left(x_{0}^{\prime}\right)$ exists and is equal to 0 . Suppose that $f^{\prime}$ is of class $C^{k}$ at $x_{0}$ and $D^{i} f^{\prime}\left(x_{0}^{\prime}\right)=0$ for $1 \leq i \leq k$. With the same local coordinates systems as in Lemma 3.3, for any $k \in \mathbb{N}$, we have 


$$
\lim _{n \rightarrow \infty} \frac{D^{k} f^{\prime}\left(z_{n}^{\prime}\right)-D^{k} f^{\prime}(0)}{\left|z_{n}^{\prime}\right|}=\lim _{n \rightarrow \infty} \frac{D^{k} f\left(z_{n}\right)}{\left|z_{n}\right|^{m}}=0 .
$$

This shows that this limit exists and is equal to $\lim _{n \rightarrow \infty} D^{k} f^{\prime}\left(z_{n}^{\prime}\right)=0$ which shows that $D^{k+1} f^{\prime}$ is continuous at $x^{\prime}=\pi(x)$ and equal to 0 .

Let $\pi: U \subset S \rightarrow M^{\prime}$ be a $C^{1}$-map. We have the following well-known formula for the gradient of the composition

$$
\nabla(f \pi)(z):=(D \pi)^{*}(z)[\nabla(f)(\pi(z))] \in T_{z} S,
$$

where the latter is the evaluation of the matrix field $(D \pi)^{*}(z)$ on the vector field $\nabla(f)(\pi(z))$ and $(D \pi)^{*}(z): T_{\pi(z)} M^{\prime} \rightarrow T_{z} M$ is the linear operator conjugate (dual) to the linear map $D \pi(z)$.

Let $U \subset M, V \subset M^{\prime}$ be domains of local charts identified with subsets of $\mathbb{C}$, e.g. $U$ is a disc with the centre at 0 . Next, let $\pi(z)=z^{m},|m| \geq 1$ be a map from $U$ into $V$. Then, $(D \pi)^{*}(z)=\left(D\left(z^{m_{i}}\right)\right)^{*}(z)=m\left(z^{m-1}\right)^{*}=m \bar{z}^{m-1}$. This remark leads to the next Lemma.

Let $U, V$ be two open subsets of $\mathbb{C}$ such that $0 \in U \cap V$ and let $\pi: U \rightarrow V, \pi$ : $(U, U \backslash\{0\}) \rightarrow(V, V \backslash\{0\})$ be an orientation-preserving $C^{1}$-map such that $D \pi(0)=0$ and $D \pi(z) \neq 0$ if $z \neq 0$. Let also $f^{\prime}: V \rightarrow \mathbb{R}$ be a $C^{1}$-function with isolated critical or regular point at 0 . By deg we shall denote the topological degree of maps $\nabla(f)$ and $\pi$ with respect to the pair $\left(\mathbb{D}_{\varepsilon}, \mathbb{D}_{\varepsilon} \backslash\{0\}\right) \rightarrow(\mathbb{C}, \mathbb{C} \backslash\{0\})$, where $\mathbb{D}_{\varepsilon} \subset \mathbb{C}$ is a small disc centered at 0 . Observe that $\operatorname{deg}(\nabla(f), 0)=0$ if 0 is not a critical point of $f$. Next, denote by $\operatorname{deg}\left(D \pi^{*}, 0\right)$ the degree of $D \pi^{*}: S_{\varepsilon}^{1} \rightarrow \operatorname{GL}(2, \mathbb{R})$. Notice that each of two connected components of $\operatorname{GL}(2, \mathbb{R})$ is homotopically equivalent to $\mathbb{S}^{1}$, since $\mathrm{GL}(2, \mathbb{R})_{+} \equiv \mathrm{O}(2)_{+}=\mathrm{SO}(2)=\mathbb{S}^{1}$. In other words, this degree is an element of $\pi_{1}(\mathrm{GL}(2, \mathbb{R}))=\mathbb{Z}$.

Observe that in our case $\pi$ is a holomorphic map. Since $D \pi(z) \neq 0$ for $z \neq 0$ and 0 is the unique critical point of $f^{\prime}, 0$ is also the unique critical point of the composition $f=$ $f^{\prime} \pi: V \rightarrow \mathbb{R}$ and therefore $\nabla\left(f^{\prime} \pi\right)(z)$ is a map of pairs $(V, V \backslash 0) \rightarrow(\mathbb{C}, \mathbb{C} \backslash\{0\})$.

Lemma 3.5 Under the above assumptions

$$
\operatorname{deg}\left(\nabla\left(f^{\prime} \pi\right), 0\right)=\operatorname{deg}(\pi, 0) \operatorname{deg}\left(\nabla\left(f^{\prime}\right), 0\right)+\operatorname{deg}\left(D \pi^{*}, 0\right) .
$$

Proof We already know that

$$
\nabla\left(f^{\prime} \pi\right)(z)=(D \pi)^{*}(z)\left[\nabla\left(f^{\prime}\right)(\pi(z))\right]
$$

where the latter is the evaluation of the matrix field $(D \pi)^{*}(z)$ on the vector field $\nabla\left(f^{\prime}\right)(\pi(z))$. Since $\pi$ is holomorphic, $D \pi(z)$ is the multiplication by the complex derivative of the map $\pi$. Applying Lemma 3.6,

$$
\begin{aligned}
\operatorname{deg}\left((D \pi)^{*}(z)\left[\nabla\left(f^{\prime}\right)(\pi(z))\right]\right) & =\operatorname{deg}\left((D \pi)^{*}(z)\right)+\operatorname{deg}\left(\nabla\left(f^{\prime}\right)(\pi(z))\right) \\
& =\operatorname{deg}\left((D \pi)^{*}(z)\right)+\operatorname{deg}\left(\left[\nabla\left(f^{\prime}\right)\right]\right) \cdot \operatorname{deg}(\pi(z)) .
\end{aligned}
$$


It remains to prove the following lemma.

Lemma 3.6 Let $U \subset \mathbb{C}$ be an open subset, $\alpha, \beta: U \rightarrow \mathbb{C}$ continuous maps with $\alpha^{-1}(0) \cup \beta^{-1}(0)$ compact and let $(\alpha * \beta)(z)=\alpha(z) \cdot \beta(z)$, where the last is the product of complex numbers. Then,

$$
\operatorname{deg}(\alpha * \beta)=\operatorname{deg} \alpha+\operatorname{deg} \beta
$$

Proof We assume that $U$ is connected. One can find homotopies with compact support, making the sets $\alpha^{-1}(0), \beta^{-1}(0)$ disjoint, finite and consisting of regular points only; here, the degrees do not change. Let us denote $\alpha^{-1}(0)=\left\{x_{1}, \ldots, x_{k}\right\}, \beta^{-1}(0)=$ $\left\{y_{1}, \ldots, y_{l}\right\}$. Obviously $(\alpha * \beta)^{-1}(0)=\left\{x_{1}, \ldots, x_{k}\right\} \cup\left\{y_{1}, \ldots, y_{l}\right\}$. By the definition of the degree, $\operatorname{deg}(\alpha * \beta)$ is equal to

$$
\operatorname{deg}(\alpha * \beta)=\sum \operatorname{deg}(\alpha * \beta ; z)=\sum_{i=1}^{k} \operatorname{deg}\left(\alpha * \beta ; x_{i}\right)+\sum_{j=1}^{l} \operatorname{deg}\left(\alpha * \beta ; y_{j}\right)
$$

where the sum is taken over $z \in(\alpha * \beta)^{-1}(0)$. Consider a point $x_{i}$. Since $\beta(z) \neq 0$ near $x_{i}, \operatorname{deg}\left(\alpha * \beta ; x_{i}\right)=\operatorname{deg}\left(\alpha ; x_{i}\right)$ and similarly $\operatorname{deg}\left(\alpha * \beta ; y_{i}\right)=\operatorname{deg}\left(\beta ; y_{j}\right)$ for all $j=1, \ldots, l$. By $(2)$,

$$
\operatorname{deg}(\alpha * \beta)=\sum_{i=1}^{k} \operatorname{deg}\left(\alpha ; x_{i}\right)+\sum_{j=1}^{l} \operatorname{deg}\left(\beta ; y_{j}\right)=\operatorname{deg}(\alpha)+\operatorname{deg}(\beta)
$$

Corollary 3.7 Let $f^{\prime}: V \rightarrow \mathbb{R}, 0 \in V$, be a $C^{1}$-map as in Lemma 3.5 with $\operatorname{deg}\left(\nabla\left(f^{\prime}\right), 0\right)=d$ and $\pi(z)=z^{m}$. Then, 0 is a critical point of $f:=f^{\prime} \pi$, $\operatorname{deg}(\nabla(f), 0)$ is well defined and we have

$$
\operatorname{deg}(\nabla(f), 0)=d m+1-m .
$$

Proof Note that $D \pi(z)=m z^{m-1}$ and thus the map $z \mapsto D \pi(z)$ is homotopic to the map $z \mapsto z^{m-1}$, i.e. $\operatorname{deg}\left((D \pi)^{*}, 0\right)=-(m-1)$. Hence, the result follows by the Lemma 3.5.

\subsection{A differential proof of the Riemann-Hurwitz formula}

In the last section, we present a new proof of the Riemann-Hurwitz formula for a Riemann surface $\mathcal{S}$. It is completely elementary and requires only the existence of a $C^{1}$-function with all isolated (thus finite in number) critical points on $S$ and Hopf formula for the gradient field of such a function. It is similar to that of [15], but we do not assume that our function is Morse and allow its critical points to be in the image, by $\pi: \mathcal{S} \rightarrow \mathcal{S}^{\prime}$, of the singular points set of the action of $G$ on $\mathcal{S}$ (see [15] for references about other proofs of the Riemann-Hurwitz formula). 
Let $\mathcal{S}$ be a compact Riemann surface of genus g $>1$ and let $G$ be a group of holomorphic automorphisms of $\mathcal{S}$. Let $\mathcal{S}^{\prime}=\mathcal{S} / G$ be the quotient surface of genus $\mathrm{g}^{\prime}$ with the projection $\pi: \mathcal{S} \rightarrow \mathcal{S}^{\prime}$ and let $\left\{x_{1}^{\prime}, \ldots, x_{r}^{\prime}\right\}$ be the set of all points over which $\pi$ is branched, i.e. the image of the singular orbits. For any $x_{i, j} \in \pi^{-1}\left(x_{j}^{\prime}\right)$ being a point in the orbit over $x_{j}^{\prime}$, the isotropy group $G_{x_{i, j}}$ is cyclic of order $m_{j}$ (observe that the isotropy groups of points in one orbit are conjugate). We denote by $m$ the order of $G$. Now, it follows that the orbit of $x_{i, j}$ has order $r_{j}=m / m_{j}$. If $x^{\prime} \in \mathcal{S}^{\prime} \backslash\left\{x_{1}^{\prime}, \ldots, x_{r}^{\prime}\right\}$, then $\pi^{-1}\left(x^{\prime}\right)$ is an orbit isomorphic to $G$ and so consequently, for every $x \in \pi^{-1}\left(x^{\prime}\right)$, $m_{x}=1$ and $r_{x}=m / m_{x}=m$.

We already know, that given a $C^{1}$ and $G$-invariant function $f: \mathcal{S} \rightarrow \mathbb{R}$, with isolated critical points, every singular point $x \in \mathcal{S}$ is a critical point of $f$, i.e.

$$
\bigcup_{j=1}^{r} \bigcup_{i=1}^{r_{j}}\left\{x_{i, j}\right\} \subset \operatorname{Crit}(f) .
$$

Theorem 3.8 Let $f: \mathcal{S} \rightarrow \mathbb{R}$ be a $C^{1}$ and $G$-invariant function with isolated critical points satisfying the condition (3) of Theorem 3.2 at every singular point of the action. Then, $f$ induces a $C^{1}$-function $f^{\prime}: \mathcal{S}^{\prime} \rightarrow \mathbb{R}$ on the orbit space $\mathcal{S}^{\prime}$ with

$$
\operatorname{Crit}\left(f^{\prime}\right)=\pi(\operatorname{Crit}(f))=\left\{x_{1}^{\prime}, \ldots, x_{r}^{\prime}, \bar{x}_{1}^{\prime}, \ldots \bar{x}_{\bar{r}}^{\prime}\right\}, \quad \bar{r} \geq 0 .
$$

Furthermore, the local indices of the vector field $\operatorname{deg}(\nabla(f), x)$ do not depend on a choice of a representative of the orbit of $x$, i.e. do not depend on $i$ and we have the following formulas

$$
\begin{aligned}
\chi(\mathcal{S})=2-2 \mathrm{~g} & =\sum_{j=1}^{r} \sum_{i=1}^{r_{j}} \operatorname{deg}\left(\nabla(f), x_{i, j}\right)+\sum_{j=1}^{\bar{r}} \sum_{i=1}^{m} \operatorname{deg}\left(\nabla(f), \bar{x}_{i, j}\right) \\
& =\sum_{j=1}^{r} \operatorname{deg}\left(\nabla(f), x_{i_{0}, j}\right) r_{j}+\sum_{j=1}^{\bar{r}} \operatorname{deg}\left(\nabla(f), \bar{x}_{i_{1}, j}\right) m
\end{aligned}
$$

where $x_{i_{0}, j}$ and $\bar{x}_{i_{1}, j}$ are some fixed points in the orbits over $x_{j}^{\prime}$ and $\bar{x}_{j}^{\prime}$, respectively.

$$
\chi\left(\mathcal{S}^{\prime}\right)=2-2 \mathrm{~g}^{\prime}=\sum_{j=1}^{r} \operatorname{deg}\left(\nabla\left(f^{\prime}\right), x_{j}^{\prime}\right)+\sum_{j=1}^{\bar{r}} \operatorname{deg}\left(\nabla\left(f^{\prime}\right), \bar{x}_{j}^{\prime}\right)
$$

Lemma 3.9 Let $\mathfrak{S}(x)=\nabla(f)(x)$ be a vector field defined on a small disc $\mathbb{D}_{\varepsilon}\left(x_{0}\right)$ near a critical point $x_{0}$ of $f$. Then, $x_{0}^{\prime}=\pi\left(x_{0}\right)$ is a critical point of $f^{\prime}=f / G$ and we have

$$
\operatorname{deg}\left(\nabla(f), x_{0}\right)=\operatorname{deg}\left(\nabla\left(f^{\prime}\right), x_{0}^{\prime}\right)
$$




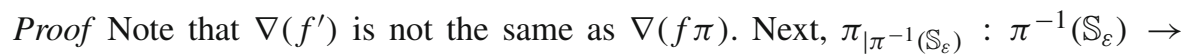
$\overline{\mathbb{S}}_{\varepsilon} / G$ is a regular covering of multiplicity $m$. Moreover, $\pi$ is an orientation-preserving diffeomorphism on each of $m$-connected components of this set and thus $\nabla(f)(x)=$ $\nabla\left(f^{\prime}\right)(\pi(x))$ on $\mathbb{S}_{\varepsilon}\left(x_{0}\right)$. Furthermore, due to this identification, $\operatorname{deg}\left(\nabla(f), x_{0}\right)=$ $\operatorname{deg}\left(\nabla\left(f^{\prime}\right), x_{0}^{\prime}\right)$, where $x_{0}^{\prime}=\pi\left(x_{0}\right)$.

Let us introduce the following notation. For $1 \leq j \leq r$, let $d_{j}$ denote the degree $\operatorname{deg}\left(\nabla(f), x_{i, j}\right)$, where for $1 \leq j \leq r, x_{i, j} \in \pi^{-1}\left(x_{j}^{\prime}\right)_{i=1}^{r_{i}}$ is any point of the orbit. Recall that this degree does not depend on $i$. Similarly, for $1 \leq j \leq \bar{r}$ let $\bar{d}_{j}$ denote the degree $\operatorname{deg}\left(\nabla(f), \bar{x}_{i, j}\right)$, where for $1 \leq j \leq \bar{r}, \bar{x}_{i, j} \in \pi^{-1}\left(\bar{x}_{j}^{\prime}\right)_{i=1}^{m}$ is any point of the orbit; again this degree does not depend on $i$.

Next, for $1 \leq j \leq r$ and $1 \leq j \leq \bar{r}$ let $d_{j}^{\prime}$, or correspondingly $\bar{d}_{j}^{\prime}$, denote $\left.\operatorname{deg}\left(\nabla\left(f^{\prime}\right), x_{j}^{\prime}\right)\right)$, or $\operatorname{deg}\left(\nabla\left(f^{\prime}\right), \bar{x}_{j}^{\prime}\right)$, respectively. Hence, by Lemma 3.9, we have

$$
\text { for every } 1 \leq j \leq r \text {, and } 1 \leq j \leq \bar{r} \text { we have } d_{j}=d_{j}^{\prime}, \bar{d}_{j}=\bar{d}_{j}^{\prime} .
$$

Then, the second equality of Theorem 3.8 has the form

$$
2-2 \mathrm{~g}^{\prime}=\sum_{j=1}^{r} d_{j}^{\prime}+\sum_{j=1}^{\bar{r}} \bar{d}_{j}^{\prime}
$$

Now, using the Euler-Poincare formula for $\mathfrak{S}(x)=\nabla(f \pi)(x)$ and the statement of Corollary 3.7, we have

$$
\begin{aligned}
\chi(\mathcal{S}) & =2-2 \mathrm{~g} \\
& =\sum_{j=1}^{r} \sum_{i=1}^{r_{j}}\left(m_{j} d_{j}+1-m_{j}\right)+\sum_{j=1}^{\bar{r}} \sum_{i=1}^{m} \bar{d}_{i, j} \\
& =\sum_{j=1}^{r} r_{j}\left(m_{j} d_{j}^{\prime}+1+m_{j}\right)+\sum_{j=1}^{\bar{r}} m \bar{d}_{j}^{\prime}
\end{aligned}
$$

Comparing the Eqs. (3), (4) and $r_{j}=m / m_{j}$ we get

$$
\mathrm{g}=1+m\left(\mathrm{~g}^{\prime}-1\right)+\frac{1}{2} m \sum_{j=1}^{r}\left(1-\frac{1}{m_{j}}\right)
$$

which is the classical Riemann-Hurwitz formula.

\section{The computation of equivariant category of surface}

The equivariant category of a preserving orientation action of finite group on closed orientable surface will be calculated in several steps. 


\subsection{Basic filtration}

We begin with some general statement on the equivariant Lusternik-Schnirelmann category. The question of a computation of equivariant category for a topological space $X$ is of interest, but difficult in general. However, there is a direct, well-known estimate for it, by the category of the orbit space [11].

Proposition $4.1 \operatorname{cat}_{G}(A ; X) \geq \operatorname{cat}(A / G ; X / G)$.

We shall use this standard lower bound for the $G$-category of $X$ and an upper bound for it given in [4]. To formulate the latter, we have to present an idea of the standard filtration of a $G$-space. Colman [4] introduced and explored a notion of basic filtration of a $G$-space but in our case of $G$ being finite these two terms coincide.

Any homogenous space $G / H$ is uniquely determined by the conjugacy class $(H)$ of $H$. Moreover, the orbit of any point $x \in X$ is isomorphic, as a $G$-space, to $G / G_{x}$. Given a subgroup $H \subset G$, the set of all points $x$ of $X$ for which $\left(G_{X}\right)=(H)$, denoted by $X_{(H)}$, is called the orbit type. It is an open subset of the set $X^{(H)}=G X^{H}$ of all points for which $G_{X}$ is conjugate to a subgroup of $\mathrm{g} \mathrm{Hg}^{-1}$.

From now on, we shall assume that $G$ is a finite group and its action is effective, i.e. the kernel $K$ of $\varphi: G \rightarrow \operatorname{Homeo}(X)$ is trivial. Observe that actually this is not a restriction, as then $\operatorname{cat}_{G}(X)=\operatorname{cat}_{G / K}(X)[1,11]$. If $G$ is finite and $X$ is a manifold, from the properties of finite group actions on $X$, it follows directly that:

- there are points with the isotropy group $G_{x}$ being trivial, i.e. equal to $e$;

- the set $X_{e}$ of points with $G_{x}=e$ is invariant, open, dense, and $X_{e} / G$ is connected. The set $X \backslash X_{e}$ is called, the set of singular points.

Remark 4.2 The above holds in more general situation in the following form. For a smooth action of compact Lie group, there exists an orbit type $X_{(H)}$, which is called the principal orbit type and which has the properties listed above, i.e. it is open, dense, and $X_{(H)} / G$ is connected.

A subgroup $H$ of a group $G$ will be denoted by $H \leq G$. Let $\mathfrak{H}$ be the set of all subgroups of $G$. For an effective action of a group $G$, we introduce a finite filtration of $\mathfrak{H}$ being the sequence

$$
\{e\}=\mathfrak{H}_{0} \supset \mathfrak{H}_{1} \supset \ldots \mathfrak{H}_{s}=\{G\}
$$

where

$$
\mathfrak{H}_{i+1}=\left\{H \leq G \mid \exists H_{i} \in \mathfrak{H}_{i} \text { such that } H \supsetneq H_{i}\right\}
$$

for each $0 \leq i \leq s-1$. So $H \in \mathfrak{H}_{i}$ if there is a chain of subgroups of length $l \geq i$ of the form $\{e\} \supsetneqq H_{1} \supsetneqq H_{2} \ldots \supsetneqq H_{l}=H$.

Remark 4.3 Note that if a subgroup $H$ of $G$ belongs to $\mathfrak{H}_{i}$, then every $H^{\prime} \in(H)$ also belongs to $\mathfrak{H}_{i}$. Indeed, if for $l \geq i, H^{\prime}=g^{-1} H g$ and

$$
\{e\} \supsetneqq H_{1} \supsetneqq H_{2} \supsetneqq \cdots \supsetneqq H_{l}=H
$$


is a chain for $H$, then for $H_{i}^{\prime}=g^{-1} H_{i} g$,

$$
\{e\} \supsetneqq H_{1}^{\prime} \supsetneqq H_{2}^{\prime} \supsetneqq \cdots \supsetneqq H_{l}^{\prime}=H^{\prime}
$$

is the corresponding chain for $H^{\prime}$.

To shorten notation, we denote by $B=X / G$ for the orbit space of the action of $G$ on $X$. The image of $X^{H}$ in this orbit space is denoted by $B^{H}$. Note that $B^{H}=X^{(H)} / G$. The singular set of $B$ is the image of the set of points with nontrivial isotropy in $X$, i.e. the image of the set of singular points. We will construct a filtration of the space $B$. Let

$$
X^{i}=\bigcup_{H \in \mathfrak{H}_{i}} X^{H} \text { and } B^{i}=X^{i} / G
$$

for each $i$ in the range $0 \leq i \leq s$. Observe, following Remark 4.3, that the set $X^{i}$ is $G$-invariant. Now, $B^{1}$ is the singular set and $B^{r}=B^{G}$ is the homeomorphic image of the set of points fixed by the action, possibly empty. Let also $k=\min \left\{i: X^{i+1}=\varnothing\right\}$. Finally, we introduce the basic filtration of length $k$ of $B$ to be

$$
\mathfrak{B}:=B=B^{0} \supset B^{1} \supset \cdots \supset B^{k} .
$$

Remark 4.4 Note that in our case all $X^{i}, i \geq 1$, are finite and so the corresponding $B^{i}$ are finite too. Indeed, the fixed point set $X^{H}$ is a submanifold of $X$ and as such has dimension 0 or 1 . The case of 1 -dimensional submanifold can be ommitted, since the action preserves the orientation. Moreover, for an action of holomorphic maps, such a submanifold should be complex, thus of dimension 0 .

For a $G$-space $X$, by $\mathfrak{H}(X)$ we denote the intersection of the standard filtration of all subgroups and the family of all isotropy subgroups $\left\{G_{x}: x \in X\right\}$. As a direct consequence of Proposition 2.6, we have the following fact

Proposition 4.5 If a finite group $G$ acts on a compact orientable surface $X$, then the standard filtration $\mathfrak{H}(X)$ consists only of the cyclic groups.

\subsection{Bounds for the equivariant category}

Now, we recall some lower and upper bounds for $G$-category. The lower ones are standard, while the upper one follows from the main result of [4]. From now on, we shall assume that the action is not free (for free actions the $G$-category of $X$ coincides with the category of the orbit space $X / G$, see [11]).

Proposition 4.6 $\operatorname{cat}_{G}(X) \geq \operatorname{cat}_{G}\left(X^{i}\right)$ for all $i=1, \ldots, s$.

Remark 4.7 In general, $\operatorname{cat}_{G}(A ; X) \leq \operatorname{cat}_{G}(X)=\operatorname{cat}_{G}(X ; X)$, for a $G$-invariant closed subset $A$ of $X$. But $X^{1}$ is a special invariant subset, which consists of points fixed by all nontrivial subgroups of $G$. By the definition, $X^{1}$ consists of the fixed points 
of minimal nontrivial, i.e. cyclic of a prime order, subgroups but it also contains points fixed by all the other subgroups, since $X^{H} \subset X^{K}$ for $K \subset H$.

Corollary $4.8 \operatorname{cat}_{G}(X) \geq \operatorname{cat}\left(B^{i}\right)$ for all $i=1, \ldots, s$.

Below we recall an upper bound for the $G$-category, in terms of the dimension of $B=X / G$ and the $G$-category of the singular set given by Colman [4].

Theorem 4.9 Let $X$ be a connected $G$-complex, where $G$ is a finite group acting non-freely on $X$. Then, $\operatorname{cat}_{G}(X) \leq \operatorname{dim} B+\operatorname{cat}_{G}\left(X^{1}\right)$.

Our aim is to show that for an orientation-preserving action of a finite group $G$ on a closed, connected, orientable surface $X$ of genus $g \geq 2$, the $G$-category is realized on the set of singular orbits in vast majority cases. In Example 4.4, in [4], we have an action of $G=\mathbb{Z}_{4}$ on the torus, for which

$$
\operatorname{cat}_{\mathbb{Z}_{4}}\left(\mathbb{T}^{2}\right)=3=\operatorname{cat}_{\mathbb{Z}_{4}}\left(X^{1}\right)
$$

Here, the quotient surface $X^{\prime}$ is homeomorphic to $\mathbb{S}^{2}$.

We begin with the following lemma.

Lemma 4.10 Let $M^{\prime}$ be a compact orientable two-dimensional closed manifold, of genus $\mathrm{g}$ and let $x_{1}^{\prime}, \ldots, x_{r}^{\prime}$ be a finite set of points of $M^{\prime}$. Then, there exists a $C^{1}$ function $f^{\prime}: M^{\prime} \rightarrow \mathbb{R}$ such that all the critical points of $f^{\prime}$ are:

(a) $x_{1}^{\prime}, x_{2}^{\prime}$ if $\mathrm{g}=0$ and $r \geq 2$,

(b) $x_{1}^{\prime}, x_{2}^{\prime}, x_{3}^{\prime}$ if $\mathrm{g} \geq 1$ and $r \geq 3$,

(c) $x_{1}^{\prime}$ and another $\tilde{x}_{2}^{\prime}$ if $\mathrm{g}=0$ and $r=1$,

(d) $x_{1}^{\prime}$ and another two points $\tilde{x}_{2}^{\prime}, \tilde{x}_{3}^{\prime}$ or $x_{1}^{\prime}, x_{2}^{\prime}$ and another $\tilde{x}_{3}^{\prime}$ if $\mathrm{g} \geq 1$ and $r=1$ or $r=2$, respectively.

Proof For every compact closed surface two-dimensional manifold $M$ of genus $g>0$ there exists a $C^{1}$-function $\tilde{f}: M \rightarrow \mathbb{R}$ such that $\tilde{f}$ has only three critical points (cf. [13, p. 90] and [16]), which we denote by $y_{1}, y_{2}, y_{3}$. If $M$ has genus 0 , i.e. when it is diffeomorphic to the sphere $\mathbb{S}^{2}$, then there exists a function $\tilde{f}: \mathbb{S}^{2} \rightarrow \mathbb{R}$ with only two critical points, denoted by $y_{1}, y_{2}$.

Next, for every three pairs of points $x_{1}^{\prime}, x_{2}^{\prime}, x_{3}^{\prime}$ and $y_{1}, y_{2}, y_{3}$ of a compact surface $M$, there exists an isotopy $\varphi_{t}: M \times I \rightarrow M$ such that $\varphi_{0}=\operatorname{id}_{S}$ and $\varphi_{1}\left(x_{i}^{\prime}\right)=y_{i}$ for $i=1,2,3$. Put $\varphi:=\varphi_{1}$. Then, the function $f^{\prime}=\tilde{f} \circ \varphi$ has critical points $x_{1}^{\prime}, x_{2}^{\prime}, x_{3}^{\prime}$ and they are the only critical points, because $\nabla\left(f^{\prime}\right)(x)=\nabla(\tilde{f})(\varphi(x)) \circ D \varphi(x)$.

Theorem 4.11 Let $X$ be a compact orientable surface with an orientation-preserving action of a finite group $G$ given through orientation-preserving homeomorphisms. Let $X^{\prime}$ be the orbit space of this action and let $\mathrm{g}^{\prime}$ be its genus. Then, the set of all points fixed by all nontrivial subgroups of $G$ is finite, which means that $X^{1}$ is either the union of $r \geq 1$ orbits or empty and we have:

$$
\operatorname{cat}_{G}(X)=\operatorname{cat}(X / G)=3 \text { and } \mathrm{g}^{\prime}>0
$$


if the action is free and

$$
\operatorname{cat}_{G}(X)=\left\{\begin{array}{l}
r \quad \text { if } r \geq 3, \text { or if } r=2 \text { and } g^{\prime}=0, \\
3 \quad \text { if } r \leq 2 \text { and } \mathrm{g}^{\prime} \geq 1
\end{array}\right.
$$

otherwise. Moreover, if the action is not free and $r \geq 3$ or $r=2$ and $\mathrm{g}^{\prime}=0$, then $\operatorname{cat}_{G}(X)=\operatorname{cat}_{G}\left(X^{1}\right)=\left|X^{1} / G\right|=\left|B^{1}\right|$.

Proof Due to the Hurwitz theorem and before-mentioned Edmonds Theorem 2.4, every such action is equivalent to a holomorphic action, which simply means that every finite group of orientation-preserving homeomorphisms of $X$ can be seen as a group of holomorphic automorphisms of a Riemann surface $\mathcal{S}$ homeomorphic to $X$. Then, the fixed point set of any subgroup of $G$ is finite (possibly empty) as a complex submanifold of $\mathcal{S}$.

First, we consider the free action case. Observe that every preserving orientation homeomorphism of $\mathbb{S}^{2}$ has a fixed point, as follows from the Lefschetz fixed point formula. It excludes $\mathrm{g}=0$. Next, if $\mathrm{g}^{\prime}=0$ then $\chi(\mathcal{S})=2-2 \mathrm{~g}=2 \mathrm{~m}$ where $m$ is the multiplicity of regular covering $p: \mathcal{S} \rightarrow \mathcal{S}^{\prime}$, since $\chi\left(\mathbb{S}^{2}\right)=2$. The latter is impossible, since $\chi(\mathcal{S}) \leq 0$, which shows that $\mathrm{g}^{\prime} \geq 1$, thus proves the first part of the statement.

Moreover for a group $G$ of holomorphic automorphisms of a compact Riemann surface $\mathcal{S}$ of genus $\mathrm{g} \geq 0$, the projection map $\pi: \mathcal{S} \rightarrow \mathcal{S}^{\prime}=\mathcal{S} / G$ onto the orbit space is a covering ramified over $r$ points, say with the ramification indices $m_{1}, \ldots, m_{r}$, and the genus of $\mathcal{S}^{\prime}$ equals $\mathrm{g}^{\prime}$. Observe that the action is free if and only if $r=0$.

Let us consider the general case of ramified covering. If $r \geq 3$ then the statement follows from Lemma 4.10. If $\mathrm{g}^{\prime}=0$, i.e. $\mathcal{S}^{\prime}=\mathbb{S}^{2}$ then, it is easy to show that for any $\mathcal{S}$, of $\mathrm{g} \geq 1$, there is not a branched covering $p: \mathcal{S} \rightarrow \mathcal{S}^{\prime}$ with $r \leq 2$. Indeed, from the Riemann-Hurwitz formula, we have

$$
\chi(\mathcal{S})=m\left(\chi\left(\mathcal{S}^{\prime}\right)-r\right)+m / m_{1}+\cdots m / m_{r} .
$$

Consequently, for $\chi\left(\mathcal{S}^{\prime}\right)=2, r=0,1,2$ is excluded if $\chi(\mathcal{S}) \leq 0$, which proves the first item of this case.

If $f: M \rightarrow \mathbb{R}$ is $G$-invariant $C^{1}$-function on a $G$-manifold $M$ and $x$ its critical point then $g x$ is also critical, i.e. the orbit of $x$ consists of critical points. Let $\left|\mathrm{Crit}_{o r}(f)\right|$ be the number of critical orbits of $f$. The main property of $G$-category says that for every $G$-invariant $C^{1}$-function

$$
\operatorname{cat}_{G}(S) \leq\left|\operatorname{Crit}_{\text {or }}(f)\right|
$$

(cf. [11]).

To prove the statement, it is enough to construct a $G$-invariant $C^{1}$-function $f$ : $\mathcal{S} \rightarrow \mathbb{R}$, with exactly as many critical orbits as it is given in the statement.

Let $\left\{x_{1}, \ldots, x_{r}\right\}$ be a complete set of representatives of all orbits and let $x_{i}^{\prime}=\pi\left(x_{i}\right)$ be the set of their images in the orbit space $\mathcal{S}^{\prime}=\mathcal{S} / G$. Next, let $f^{\prime}: \mathcal{S}^{\prime} \rightarrow \mathbb{R}$ be the function from the Lemma 4.10 and consider a composition map $f=f^{\prime} \pi$. By the 
definition, $f$ is $G$-invariant. By Theorem 3.2, $f$ is a $C^{1}$-function and if $x^{\prime}=\pi(x)$ is a regular point of $f^{\prime}$ then $x$ is a regular point of $f$. Furthermore, every point $x \in G x_{i}$, $1 \leq i \leq r$ of a singular orbit of action is a critical point of $f$, by (1) of Theorem 3.2. Finally, if $x^{\prime}, x^{\prime}=\pi(x)$ is a critical point of $f^{\prime}$, then the orbit of $x$ consists of critical points of $f$.

Summing up, the set of the critical points of $f$ consists of all points in $G x_{1} \cup \ldots \cup G x_{r}$ and points (thus orbits) which are not in this set but which are mapped by $\pi$ onto critical points of $f^{\prime}$. The latter corresponds to the cases (c) and (d) of the Lemma 4.10.

To complete the proof of Theorem 4.11, observe that $\operatorname{cat}\left(B^{1}\right)=|B|$ is equal to cat ${ }_{G}\left(\mathcal{S}^{1}\right)$, because $B^{1}$ is the finite union of points corresponding to the orbits of the $G$-set $X^{1}$. Consequently, cat $\left(B^{1}\right)=\left|B^{1}\right|$. Since $\mathcal{S}^{1}$ consists of $r$ orbits, we get the claim

$$
\operatorname{cat}_{G}\left(X^{1}\right)=\operatorname{cat}_{G}\left(\mathcal{S}^{1}\right)=\operatorname{cat}\left(B^{1}\right)=\left|B^{1}\right|=r
$$

Remark 4.12 Note that for every $m$ there exists a semi-free preserving orientation action of $G=\mathbb{Z}_{m}$ with two fixed points (the rotation of the sphere around its axis by $\frac{2 \pi}{m}$ angle). Also, for every $g \geq 1$, there exits a branching cover of a Riemann surface $\mathcal{S}$ of genus g over $\mathcal{S}^{\prime}=\mathbb{S}^{2}$ with exactly three branching points.

In the examples given below, we use a representation of a Riemann surface $\mathcal{S}$ as a quotient of the upper half-plane $\mathcal{H}$, with the hyperbolic metric, by a discrete group $\Gamma$ of its isometries, called the Fuchsian group. The group $G$ acting on $\mathcal{S}$ is by biholomorphic maps defined by a supgroup $\Lambda \supset \Gamma$ of isometries in which $\Gamma$ is a normal subgroup and $\Lambda / \Gamma=G$ (see [2] for more details).

Example 4.13 Given $h>0$, there exists a Fuchsian group $\Lambda$ with the presentation

$$
\left\langle x_{1}, a_{1}, b_{1}, \ldots, a_{h} b_{\mathrm{h}} \mid x_{1}^{3}=1,\left[a_{1}, b_{1}\right] \ldots\left[a_{\mathrm{h}}, b_{\mathrm{h}}\right] x_{1}=1\right\rangle .
$$

Let

$$
\theta: \Lambda \rightarrow G=\mathrm{D}_{3}=\left\langle a, b \mid a^{2}, b^{2},(a b)^{3}\right\rangle
$$

be defined by $\theta\left(x_{1}\right)=a b, \theta\left(a_{i}\right)=a$ for $i=1, \ldots \mathrm{h}, \theta\left(b_{i}\right)=b$ for $i=1, \ldots, \mathrm{h}-2$ and let $\left(b_{\mathrm{h}-1}, b_{\mathrm{h}}\right)$ be mapped to

$$
\begin{array}{lll}
(b, b a b) & \text { for } h \equiv 0 & \bmod 3 \\
(b, b) & \text { for } h \equiv 1 & \bmod 3 \\
(b a b, b a b) & \text { for } h \equiv 2 & \bmod 3
\end{array}
$$

Then, for $\Gamma=\operatorname{ker} \theta$, the orbit space $\mathcal{S}^{\prime}=\mathcal{H} / \Gamma$, where $\mathcal{H}$ is the hyperbolic plane is a Riemann surface with a group of automorphisms $G=\Lambda / \Gamma$ so that $\mathcal{S}^{\prime}=\mathcal{S} / G \cong \mathcal{H} / \Lambda$ and the canonical projection $\mathcal{S} \rightarrow \mathcal{S}^{\prime}=\mathcal{S} / G$ is sixfold covering ramified over exactly 
one point. The surface $\mathcal{S}^{\prime}$ has genus h and, by the Hurwitz-Riemann formula $\mathcal{S}$ has genus $g=3(2 \mathrm{~h}-1)$.

Observe that the above constructed covering is extremal in the sense that $g$ is the minimal number for the genus of a Riemann surface which allow a covering of Riemann surface ramified over one point.

Observe also that 6 is the minimal degree for such covering since each group of order smaller than 6 is abelian, while for abelian group $G$ the long relation in $\Lambda$ is mapped by arbitrary epimorphism $\theta$ into $\theta\left(x_{1}\right)$ and therefore $x_{1}$ is a torsion element of $\Gamma$ in contrary to our assumption.

Example 4.14 Given $\mathrm{h}>0$, there exists a Fuchsian group $\Lambda$ with the presentation

$$
\left\langle x_{1}, x_{2}, a_{1}, b_{1}, \ldots, a_{\mathrm{h}}, b_{\mathrm{h}} \mid x_{1}^{2}=1, x_{2}^{2}=1,\left[a_{1}, b_{1}\right] \ldots\left[a_{\mathrm{h}}, b_{\mathrm{h}}\right] x_{1} x_{2}=1\right\rangle
$$

and let

$$
\theta: \Lambda \rightarrow G=\mathbb{Z}_{2}=\langle a\rangle
$$

be defined by

$$
\theta\left(x_{i}\right)=\theta\left(a_{j}\right)=\theta\left(b_{j}\right)=b, \quad i=1,2, j=1, \ldots, \mathrm{h} .
$$

Then, for $\Gamma=\operatorname{ker} \theta$, the orbit space $\mathcal{S}=\mathcal{H} / \Gamma$, where $\mathcal{H}$ is the hyperbolic plane is a Riemann surface with a group of automorphisms $G=\Lambda / \Gamma$ so that $\mathcal{S}^{\prime}=\mathcal{S} / G \cong \mathcal{H} / \Lambda$ and the canonical projection $\mathcal{S} \rightarrow \mathcal{S}^{\prime}=\mathcal{S} / G$ is a twofold covering ramified over exactly two points. The surface $\mathcal{S}^{\prime}$ has genus $\mathrm{h}$ and, by the Hurwitz-Riemann formula $\mathcal{S}$ has genus $\mathrm{g}=2 \mathrm{~h}$.

Note that in the above examples $D_{n}$ is the dihedral group associated to a polygon with $n$ sides.

Theorem 4.9 applied to a closed surface $\mathcal{S}$ gives cat $_{G}(\mathcal{S})-\left|B_{1}\right|=\operatorname{cat}_{G}(\mathcal{S})-r \leq 2$, since $\operatorname{dim} B=2$. As a consequence of Theorem 4.11 and Examples 4.13 and 4.14 we get the following

Corollary 4.15 For each of below listed cases, there exists closed oriented surface $\mathcal{S}$ of genus $g \geq 1$ with holomorphic, not free, action of finite groups $G$ such that

$\operatorname{cat}_{G}(\mathcal{S})=\left|B_{1}\right|, \quad$ or $\operatorname{cat}_{G}(\mathcal{S})=\left|B_{1}\right|+1$, or $\operatorname{cat}_{G}(\mathcal{S})=\left|B_{1}\right|+2$, respectively.

In other words, the inequality of Colman Theorem 4.9 is sharp for preserving orientation actions on surfaces. Moreover, each of three possible values for $\mathrm{cat}_{G}(\mathcal{S}):\left|B_{1}\right|$, $\left|B_{1}\right|+1$, and $\left|B_{1}\right|+2$ is attained.

Corollary 4.16 If $\mathcal{S}$ is a oriented surface of $\mathrm{g}((\mathcal{S}) \geq 1$ with a holomorphic not free action of $G$ such that $r=\left|B_{1}\right| \geq 3$ and $f: \mathcal{S} \rightarrow \mathbb{R}$, is a $C^{1} G$-invariant function 
with minimal number of critical orbits, then the set of critical orbits of $f$ is equal to the set of singular orbits of this action.

If $\mathrm{g}(\mathcal{S}) \geq 1$ and $r \leq 2$ then for every $G$-invariant function $f: \mathcal{S} \rightarrow \mathbb{R}$ there exist critical orbits of $f$ which are not singular orbits of the action.

Acknowledgments The authors would like to express their thanks to Krzysztof Pawałowski for drawing their attention to the paper of Edmonds [5] and for many helpful conversations and to both referees for their helpful remarks, comments, advices and suggestions.

Open Access This article is distributed under the terms of the Creative Commons Attribution License which permits any use, distribution, and reproduction in any medium, provided the original author(s) and the source are credited.

\section{References}

1. Bartsch, T.: Topological Methods for Variational Problems with Symmetries. Lecture Notes in Mathematics, vol. 1560. Springer, New York (1993)

2. Bujalance, E., Etayo, J.J., Gamboa, J.M., Gromadzki, G.: Automorphisms Groups of Compact Bordered Klein Surfaces. A Combinatorial Approach. Lecture Notes in Mathematics, vol. 1439. Springer, Berlin (1990)

3. Clapp, M., Puppe, D.: Critical point theory with symmetries. J. reine angew. Math. 418, 1-29 (1991)

4. Colman, H.: Equivariant LS-category for finite group actions. Lusternik-Schnirelmann category and related topics (South Hadley, MA, 2001). Contemp. Math. Am. Math. Soc. Provid. RI 316, 35-40 (2002)

5. Edmonds, A.L.: Transformation groups and low-dimensional manifolds. Group actions on manifolds (Boulder, Colo., 1983). Contemp. Math. Am. Math. Soc. Provid. RI 36, 339-366 (1985)

6. Farkas, H.M., Kra, I.: Riemann surfaces. In: Graduate Texts in Mathematics, vol. 71. Springer, New York (1992)

7. Gromadzki, G., Marzantowicz, W.: Conformal actions with prescribed periods on Riemann surfaces. Fundam. Math. 213, 169-190 (2011)

8. Hurwitz, A.: Über algebraische Gebilde mit eindeutigen Transformationen in sich. Math. Ann. 41, 402-442 (1893)

9. Harvey, W.J.: Cyclic groups of automorphisms of a compact Riemann surface. Q. J. Math. Oxf. 17, 86-97 (1966)

10. Macbeath, A.M.: Action of automorphisms of a compact Riemann surface on the first homology group. Bull. Lond. Math. Soc. 5, 103-108 (1973)

11. Marzantowicz, W.: A $G$-Lusternik-Schnirelman category of space with an action of a compact Lie group. Topology 28, 403-412 (1989)

12. Miranda, R.: Algebraic curves and Riemann surfaces. In: Graduate Studies in Mathematics, vol. 5. American Mathematical Society, Providence (1995)

13. Seifert, H., Threlfall, W.: Variationsrechnung im Grossen (Theorie von Marston Morse). Teubner, Leipzig and Berlin (1938)

14. Sierakowski, M.: Sets of periods for automorphisms of compact Riemann surfaces. J. Pure Appl. Algebra 208, 561-574 (2007)

15. Stawiska, M.: Riemann-Hurwitz formula and Morse theory, contemporary mathematics. In: PoggiCorradini, P. (ed.) p-Harmonic Equation and Recent Advances in Analysis, vol. 370, pp. 209-211 (2005)

16. Takens, F.: The minimal number of critical points of a function on a compact manifold and the Lusternik-Schnirelman category. Invent. Math. 6, 197-244 (1968)

17. Wiman, A.: Über die hyperelliptischen Kurven und diejenigen vom Geschlecht $p=3$, welche eindeutige Transformationen in sich zulassen, Bihang Till. Kongl. Svenska Vetenskaps-Akademiens Handlingar 21(1), 23 (1895) 